\title{
Proceso "steam-iron" a partir de bio-combustibles: Modelado de la etapa de reducción en lecho fijo
}

\author{
Jorge Plou, Paúl Durán, Javier Herguido, José Ángel Peña \\ Grupo de Catálisis, Separaciones Moleculares e Ingeniería de Reactores (CREG) \\ Instituto de Investigación en Ingeniería de Aragón (I3A) \\ Universidad de Zaragoza, Mariano Esquillor s/n, 50018, Zaragoza, Spain. \\ Tel. +34-976762707, e-mail: jplou@unizar.es
}

\begin{abstract}
A partir de los resultados experimentales obtenidos en reactor de lecho fijo se ha modelado y validado la reacción de reducción del proceso "steam-iron", utilizando como reactivo una mezcla sintética de composición similar a la de un bio-oil real. El modelo predice razonablemente el comportamiento del lecho de sólido en la etapa de reducción del proceso.
\end{abstract}

\section{Introducción}

Actualmente existe una gran labor de investigación en pro de los combustibles de origen biológico con el fin de limitar el uso de los combustibles fósiles, $\mathrm{y}$ por tanto reducir la amenaza del $\mathrm{CO}_{2}$ como causante del calentamiento global. El estado de agregación de estos combustibles es variado: sólido como la biomasa, líquido como el bio-oil o gaseoso como el biogás. Todos derivan de materiales de deshecho como los procedentes de la agricultura y silvicultura o de residuos urbanos. Estos se utilizan como fuente de energía primaria en procesos de obtención de combustibles de segunda generación y vectores energéticos. Entre ellos, uno de los más importantes es el hidrógeno. Éste, junto con la tecnología de pilas de combustible, tiene un gran potencial para producir energía limpia y deslocalizada que evita el aumento de contenido en gases de efecto invernadero.

El proceso "steam-iron" (SIP) (Messerschmidt 1910) consiste en ciclos de reducción y oxidación con el objetivo de producir hidrógeno de alta pureza. Como el nombre indica, es necesario utilizar óxidos de hierro, o un sólido análogo. Éste se dispone en un reactor empaquetado en el que se hace pasar una corriente reductora, como por ejemplo, bio-oil vaporizado. Tras este paso se obtiene hierro metálico, que al ser reoxidado con vapor de agua produce hidrógeno de alta pureza. Dada la naturaleza del bio-oil, es necesario trabajar a altas temperaturas, en torno a $700{ }^{\circ} \mathrm{C}$ y emplear catalizadores para favorecer su descomposición en otros componentes.

La labor experimental previa al modelado ha consistido en analizar las diferencias que existen al utilizar representantes de las diferentes fracciones presentes en el bio-oil (Bertero et al 2012; Oasmaa and Meier 2005). Entre ellos se encuentran el metanol y etanol (fracc. alcohólica), el ácido acético (fracc. ácida), la hidroxiacetona y acetona (fracc. Aldehídica/Cetónica) y finalmente agua.

Asimismo se ha procedido a estudiar el efecto de mezclas de los anteriores. Las temperaturas de operación han estado comprendidas entre 600 y 800 ${ }^{\circ} \mathrm{C}$. A partir de la observación del comportamiento de la reacción por separado de cada una de las fracciones se ha construido un modelo de reactor de lecho fijo en condiciones no estacionarias en código Matlab $^{\circledR}$. Al ser un sistema de ecuaciones en derivadas parciales en el que las concentraciones varían en el espacio y en el tiempo, requiere de un algoritmo de cálculo preciso. Para ello se ha utilizado el método de Crank-Nicolson como la mejor opción por su precisión en los resultados.

\section{Resultados}

Para resumir los resultados experimentales, se ha utilizado el diagrama de Baur-Glaessner. Los valores de $\mathrm{H}_{2} / \mathrm{H}_{2}+\mathrm{H}_{2} \mathrm{O}$ (concentración de especies) se utilizan para seguir el estado de la reacción independientemente del resto de reactivos (Fig. 1), ya que todas las especies gaseosas están vinculadas mediante el equilibrio termodinámico.

En la figura 1 están representados los valores del diagrama de varios compuestos. Se puede observar cómo se agrupan en dos pseudo-estados estacionarios: i) el equilibrio entre sólido y gas, en el que se está convirtiendo el óxido de hierro en hierro (valores de ordenada en torno a 0.75) y ii) el último (en torno a 0.93), donde se produce solo la descomposición catalítica del reactivo por falta de óxido con el que reaccionar. El tiempo está en base 
al tiempo de reacción completa teórica $\left(\mathrm{t} / \mathrm{t}_{\mathrm{teo}}\left(\mathrm{X}_{\mathrm{sol}}=1\right)\right.$.

Las conclusiones principales que se obtuvieron en los resultados experimentales fueron que independientemente del reactivo utilizado, la composición de salida es la del equilibrio termodinámico de las especies gaseosas, y que durante la reducción del sólido existe un desplazamiento constante en los valores del ratio de composición respecto del equilibrio entre el sólido y el gas.

Una vez implementadas estas conclusiones en el código en forma de equilibrios termodinámicos, acoplados al comportamiento dinámico, es posible obtener un modelo que puede ser utilizado para un escalado de un reactor de dimensiones mayores. La figura 2 muestra una comparativa entre el resultado experimental y el simulado donde se puede comprobar que la aproximación es completa durante la segunda y tercera etapas (ver zonas sombreadas en la Fig.1). El periodo correspondiente a los 7 primeros minutos está todavía en proceso de ajuste fino.

\section{REFERENCIAS}

[1]. BERTERO, M., G. DE LA PUENTE and U. SEDRAN. Fuels from bio-oils: Bio-oil production from different residual sources, characterization and thermal conditioning. Fuel [on line]. 2012, 95, 263271 [consulta: 7 mayo 2015]. Disponible en: doi:10.1016/j.fuel.2011.08.041.

[2]. MESSERSCHMITT, A. Process of producing hydrogen, U.S., Patent No. 465,686 ed. 1910.

[3]. OASMAA, A. and D. MEIER Norms and standards for fast pyrolysis liquids: 1 . Round robin test. Journal of Analytical and Applied Pyrolysis [online]. 2005, 73(2), 323-334 [consulta: 7 mayo 2015]. Disponible en: doi:10.1016/j.jaap.2005.03.003.

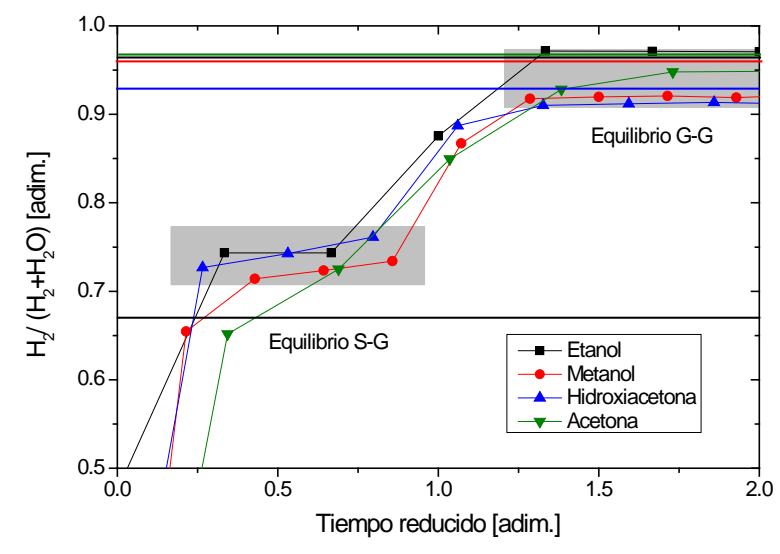

Figura 1. Ratio de $\mathrm{H}_{2}$ en la reducción a $700^{\circ} \mathrm{C}$ de varios compuestos testados

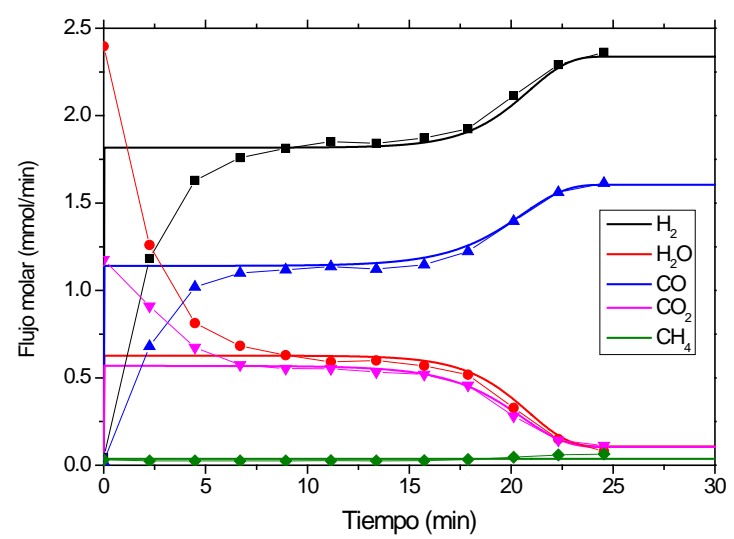

Figura 2. Datos experimentales (puntos) vs simulados (línea continua) de la reducción a $700{ }^{\circ} \mathrm{C}$ con mezcla de compuestos 